\title{
Performances de croissance des chamelons élevés dans la zone pastorale nigérienne
}

\author{
X. Pacholek ${ }^{1}$ R. Lancelot ${ }^{2}$ M. Lesnoff ${ }^{3}$ S. Messad ${ }^{3}$
}

Mots-clés

Dromadaire - Jeune animal Croissance - Mensuration corporelle Poids - Productivité - Niger.

\begin{abstract}
Résumé
De 1992 à 1996, un suivi zootechnique individuel a été réalisé sur les chamelons de moins d'un an élevés traditionnellement dans 20 troupeaux de la zone pastorale nigérienne. L'étude visait à établir une formule barymétrique simple d'estimation du poids des chamelons et à modéliser leur croissance pondérale en tenant compte d'éventuels facteurs de variation. Les données allométriques (poids vif, hauteur au garrot, périmètre thoracique) ont été anaIysées selon la méthodologie des modèles linéaires mixtes. Une formule barymétrique a été établie à partir de la mesure des périmètres thoraciques (PT) compris entre 0,7 et 1,5 $\mathrm{m}$. Le sexe est apparu comme un facteur de variation significatif $(p<0,01)$ : à périmètre thoracique égal, les femelles sont nées plus lourdes, puis le phénomène s'est inversé à partir de PT $=0,9 \mathrm{~m}$. Les poids vifs prédits ont varié entre $30,56 \pm 3,71$ et $174,02 \pm 3,01 \mathrm{~kg}$ pour les mâles et $32,37 \pm 3,67$ et $168,80 \pm 2,90 \mathrm{~kg}$ pour les femelles. Un modèle de croissance a été calculé pour les chamelons âgés de 15 à 180 jours. Aucun effet du sexe ou de la vermifugation de la mère dans les 500 jours précédant la mise bas (indicateur de l'état sanitaire et nutritionnel) n'a été mis en évidence. En revanche, les chamelons nés en saison des pluies et en début de saison sèche étaient 10 p. 100 plus lourds et croissaient 10 p. 100 plus vite que les chameIons nés en saison sèche chaude $(p<0,01)$. Les gains moyens quotidiens ont été faibles, respectivement 318 et $289 \mathrm{~g} / \mathrm{j}$ en saisons favorable et défavorable, et caractéristiques des systèmes d'élevage extensifs.
\end{abstract}

\section{INTRO DUCTION}

La première enquête d'envergure sur la productivité du cheptel camelin nigérien - estimé à 800000 têtes en 1996 (7) - a été menée de 1981 à 1983 sur 1293 troupeaux des départements de Maradi, Zinder et Diffa. Elle a dressé la typologie des élevages et a fourni de nombreuses informations sur la composition des troupeaux et leurs performances de reproduction et d'exploitation (9).

Cette enquête transversale a été complétée en 1992 par la mise en place pendant cinq ans d'un suivi longitudinal d'une trentaine de troupeaux de la zone pastorale du département de Zinder. Son objectif a été d'approfondir la connaissance des principaux facteurs de variation de la productivité des troupeaux, à partir d'un suivi individuel zootechnique et sanitaire. Cette méthodologie

1. Projet de renforcement institutionnel et technique de la filière cameline, BP 510, Niamey, Niger

Tél./Fax : +227 733607 ; E-mail : camelin@intnet.ne, xpacholek@aol.com

2. Isra/lnerv, BP 2057, Dakar-Hann, Sénégal

Tél. : +2218324902; Fax : +2218211879 ou 8323334

3. Cirad-emvt, Productions animales, Campus international de Baillarguet,

TA 30/A, 34398 Montpellier Cedex 5, France

Tél. : +330467593844; Fax : +3304675938 25 d'étude de l'élevage camelin pastoral avait été mise au point et utilisée par le Cirad-emvt au Soudan de 1989 à 1993 (1).

Un volet particulier du programme de recherche s'est intéressé aux performances de production des jeunes chamelons qui constituent le « cœur sensible » du troupeau. Cet article a eu pour objectif de présenter les principaux résultats de ces investigations en matière de croissance des chamelons, c'est-à-dire :

- l'établissement d'une formule barymétrique d'estimation du poids à partir de mesures simples comme le périmètre thoracique et la hauteur au garrot;

- la détermination d'un modèle de croissance pondérale globale et de ses facteurs de variation.

\section{MATERIEL ET METHODES}

\section{Zone de l'étude}

Le suivi a concerné toute la zone pastorale du département de Zinder situé au centre du Niger (figure 1). La zone couvrait une superficie d'environ $30000 \mathrm{~km}^{2}$. Elle recevait de juillet à septembre 250 à $350 \mathrm{~mm}$ d'eau et bénéficiait d'une végétation dominée par 


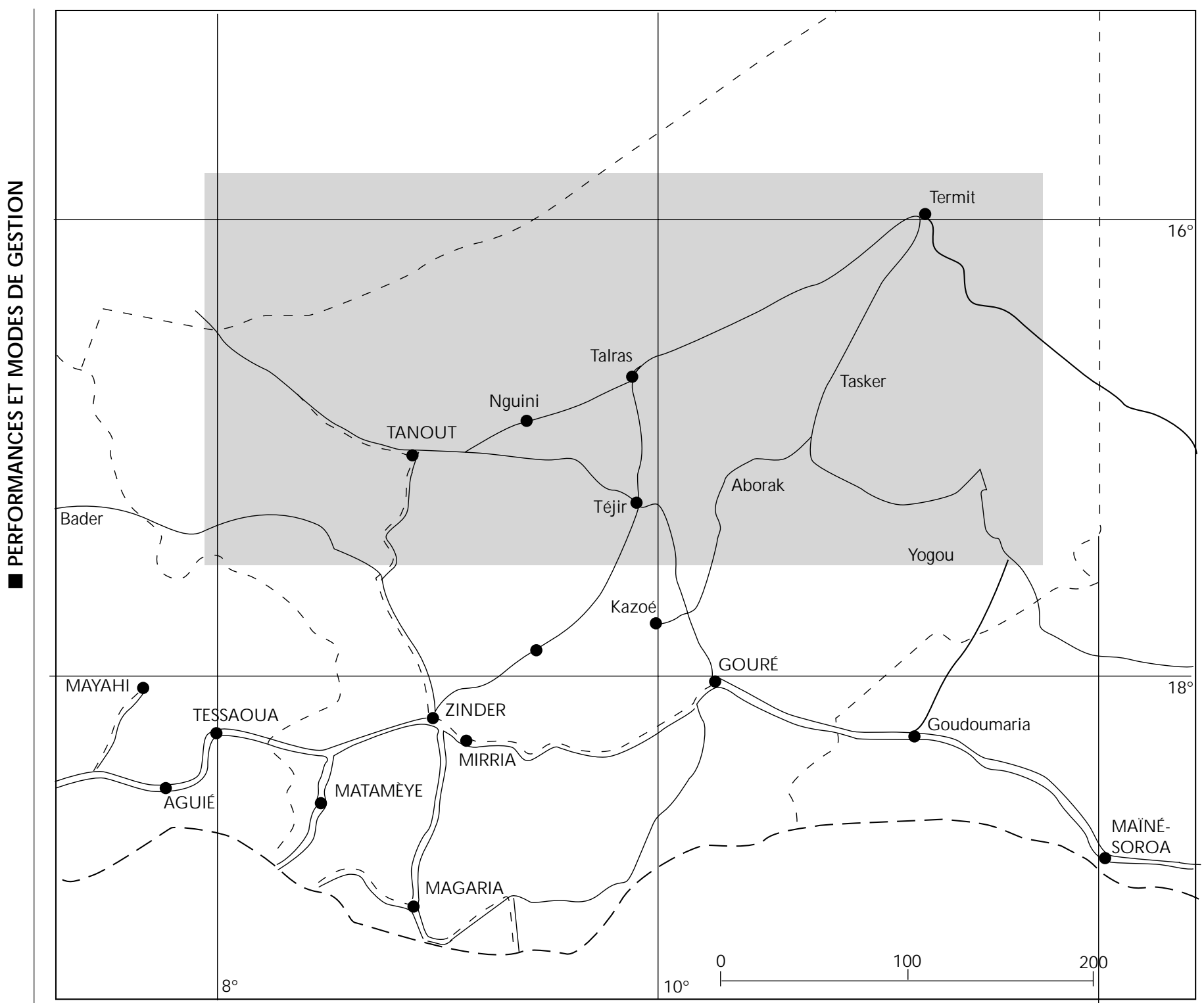

Figure 1 : localisation des troupeaux suivis.

Cenchrus biflorus et d'arbres tels que Balanites aegyptiaca, Acacia radiana et Boscia senegalensis. Dans cette zone, 85 p. 100 des éleveurs étaient Touaregs. Ils vivaient à l'est de Tasker et élevaient des dromadaires de race Azaouagh (robe claire) et Azarghaf (robe pie). A l'est, les éleveurs étaient d'ethnies Toubou et Arabes, et les dromadaires de race Manga. Globalement, les troupeaux se déplaçaient vers le nord en saison pluvieuse (migrations de moins de $100 \mathrm{~km}$ ), puis redescendaient progressivement sur leur terroir d'attache situé généralement en bordure nord des zones de culture pluviale.

\section{Echantillonnage des troupeaux}

Un échantillon raisonné d'une trentaine de troupeaux de dromadaires représentatifs de la diversité des systèmes d'élevage de la zone (9) a été intégré au suivi. Au bout des cinq années d'études, il ne restait que 20 troupeaux régulièrement visités. Leurs caractéristiques principales figurent dans le tableau I. On pourra regretter l'absence au final des quelques troupeaux Arabes initialement retenus.

\section{Suivi individuel de performances}

Dans chaque troupeau, les dromadaires étaient identifiés individuellement et de façon pérenne à l'aide d'une marque au feu, d'une boucle auriculaire ou d'une puce électronique placée sous la peau du cou et lue à l'aide d'un transpondeur. Au total, 1875 animaux ont été identifiés.

Seuls les chamelons nés pendant le suivi ont été identifiés lors des visites de troupeaux qui se sont déroulées de décembre 1992 à décembre 1996, à un rythme mensuel à bimestriel. Les informations recueillies à ces occasions étaient de nature démographique (date de naissance, date et nature de la sortie du troupeau, date et cause de la mort), sanitaire (symptômes de maladies, traitements) et zootechnique : les chamelons étaient contentionnés au bon vouloir de l'éleveur afin de mesurer leur hauteur au garrot (HG) et leur périmètre thoracique (PT) (figure 2), puis harnachés et pesés à l'aide d'un peson de $200 \mathrm{~kg}$. Les données étaient relevées sur des fiches individuelles puis validées lors de la saisie informatique sur le logiciel Pikbeu (10) qui organisait également la base de données en vue de son analyse statistique ultérieure. 
Tableau

Caractéristiques des troupeaux suivis

\begin{tabular}{|c|c|c|c|c|}
\hline $\mathbf{N}^{\circ}$ & Ethnie & Localisation & Troupeau & G ardiennage \\
\hline 1 & Touareg & Téjira & Unique & Propriétaire \\
\hline 2 & Touareg & Téjira & Unique & Propriétaire \\
\hline 3 & Touareg & Téjira & Unique & Propriétaire \\
\hline 4 & Touareg & Téjira & Unique & Propriétaire \\
\hline 6 & Toubou Aza & Bouloum & Unique & Propriétaire \\
\hline 8 & Touareg & Tenhiya & Unique & Propriétaire \\
\hline 9 & Touareg & Farak & Rassemblement & Propriétaire \\
\hline 13 & Toubou Daza & Drouanga & Divisé & Délégué \\
\hline 14 & Toubou Daza & Al Janari & Unique & Propriétaire \\
\hline 15 & Toubou Daza & Al Janari & Unique & Propriétaire \\
\hline 18 & Touareg & Belbeji & Unique & Propriétaire \\
\hline 19 & Touareg & Belbeji & Divisé & Délégué \\
\hline 20 & Touareg & Tende & Divisé & Délégué \\
\hline 21 & Touareg & Belbeji & Unique & Propriétaire \\
\hline 24 & Toubou & Bouloum & Unique & Propriétaire \\
\hline 25 & Toubou Aza & Al Janari & Rassemblement & Délégué \\
\hline 26 & Touareg & Jeptoji & Unique & Propriétaire \\
\hline 27 & Touareg & Tenhiya & Unique & Propriétaire \\
\hline 28 & Touareg & Tchintaborak & Unique & Propriétaire \\
\hline 29 & Touareg & Tchintaborak & Unique & Propriétaire \\
\hline
\end{tabular}

La contrepartie pour l'éleveur consistait en un encadrement sanitaire gratuit des animaux suivis. Cependant, les traitements vétérinaires étaient dispensés au minimum afin d'interférer le moins possible avec le suivi sanitaire.

\section{Performances de croissance}

\section{Critères d'inclusion}

Tous les chamelons entièrement suivis entre l'âge de 0 à 1 an ont été inclus. Ces chamelons ne devaient jamais avoir été malades ou traités, le poids étant influencé par l'état de santé.

\section{Méthodes d'ajustement}

Les données allométriques ont été traitées selon la méthodologie des modèles linéaires mixtes (8) et mis en œuvre avec la bibliothèque Nlme (non linear and linear mixed effects models) du logiciel S-Plus (13). Les modèles mixtes permettaient de modéliser les sources de variabilité inhérentes aux données longitudinales : variabilité individuelle liée à la répétition des mesures pour un même animal (autocorrélation) en tenant compte, en outre, de la variabilité induite par une structure agrégative des unités statistiques telle que le troupeau. Ces différents niveaux de regroupements des mesures constituaient les facteurs aléatoires du modèle.

Le modèle était estimé avec la méthode du maximum de vraisemblance restreinte (6) (Reml : restricted maximum likelihood) qui fournit des estimations non biaisées de ses termes. Le test $\mathrm{F}$ permettait de vérifier la signification des différents facteurs fixes et le test du rapport de vraisemblance (likelihood ratio) s'employait pour valider la partie aléatoire du modèle.

Les facteurs pressentis pour être testés et dont la présélection a été effectuée par les méthodes graphiques «Trellis» du logiciel
S-plus étaient le sexe, la race, le mois, la saison et l'année de naissance, les caractéristiques de l'éleveur (ethnie, localisation, propriété, gardiennage) et la vermifugation de la mère dans les 500 jours avant la mise bas (indicateur nutritionnel et sanitaire de l'état de la mère).

Après présélection graphique, les facteurs de variation retenus dans les modèles à tester étaient le sexe pour la barymétrie, le sexe, la saison de naissance (favorable de juillet à février ou défavorable de mars à juin) et la vermifugation ou non de la mère au cours des 500 jours précédant la mise bas pour la croissance pondérale.

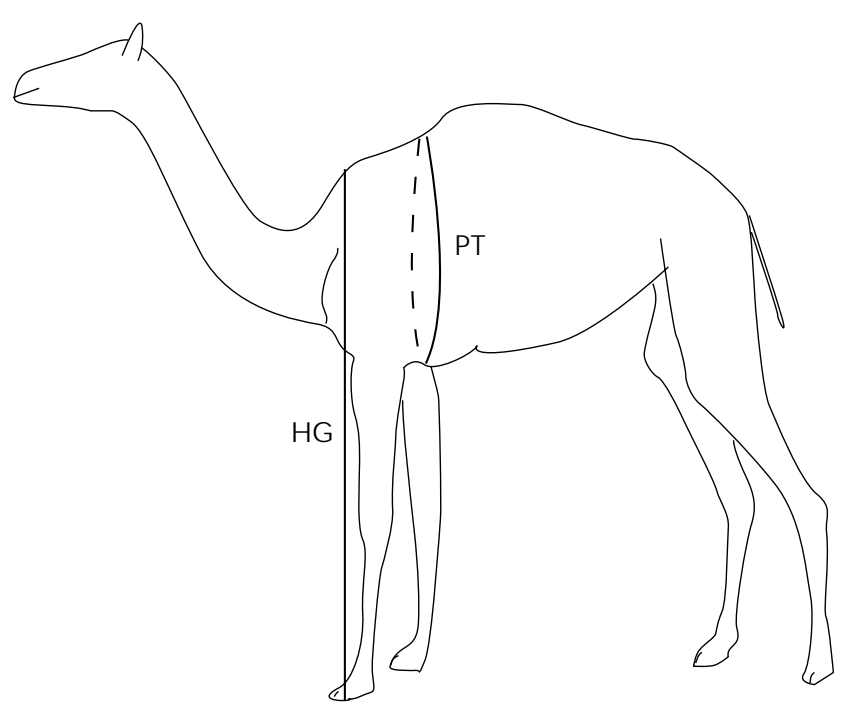

Figure 2 : hauteur au garrot et périmètre thoracique. 


\section{RESU LTATS}

\section{Formule barymétrique}

La relation allométrique linéaire $(r=0,90 ; p<0,05)$ entre HG et PT a amené à ne retenir dans le modèle que la mensuration apportant le meilleur ajustement, soit le périmètre thoracique. D'autre part, les mesures du périmètre thoracique correspondant aux queues de distribution $(<0,7 \mathrm{~m}$ et $>1,5 \mathrm{~m})$ n'ont pas été prises en compte à cause du faible nombre de mesures et de la variabilité importante du poids.
Les résultats du modèle calculés avec la méthode du maximum de vraisemblance restreinte figurent dans le tableau II. Ils révèlent une différence significative entre les mâles et les femelles $(\mathrm{p}<0,01)$.

Le tableau III et les figures 3, 4 et 5 prédisent le poids moyen des dromadaires mâles et femelles en fonction de leur périmètre thoracique. L'intervalle de confiance indiqué est applicable pour l'estimation du poids à partir de la mesure du périmètre thoracique (inférence). Il ne s'agit pas d'un intervalle de fluctuation de la courbe moyenne.

\section{Tableau II}

Estimation du modèle mixte Poids vif-Périmètre thoracique par la méthode Reml

\begin{tabular}{|c|c|c|c|c|c|c|}
\hline Paramètres & Valeur & Ecart-type & ddll 1 & ddl2 & test F & $\mathbf{p}$ \\
\hline \multicolumn{7}{|l|}{ Effets fixes } \\
\hline Constante & 94,25 & 0,43 & 1 & 1305 & 65031,13 & $<0,0001$ \\
\hline Périmètre thoracique & 187,97 & 2,08 & 1 & 1305 & 8401,94 & $<0,0001$ \\
\hline Périmètre thoracique ${ }^{2}$ & 111,33 & 8,78 & 1 & 1305 & 158,25 & $<0,0001$ \\
\hline Sexe & $-1,10$ & 0,38 & 1 & 319 & 8,42 & 0,0040 \\
\hline Périmètre thoracique $x$ Sexe & $-4,40$ & 1,95 & 1 & 1305 & 5,07 & 0,0245 \\
\hline \multicolumn{7}{|c|}{ Effets aléatoires indépendants (niveau animal) } \\
\hline $\begin{array}{l}\sigma \text { (constante) } \\
\sigma \text { (périmètre thoracique) }\end{array}$ & $\begin{array}{r}4,70 \\
14,68\end{array}$ & & & & & \\
\hline$\sigma$ (erreur résiduelle) & 9,62 & & & & & \\
\hline
\end{tabular}

$\sigma(\mathrm{a})$ : écart-type du paramètre aléatoire a

ddl1 : nombre de degrés de liberté du numérateur

ddl2 : nombre de degrés de liberté du dénominateur

Tableau III

Poids moyens ajustés par le modèle selon le périmètre thoracique et le sexe

\begin{tabular}{|c|c|c|c|c|}
\hline $\begin{array}{l}\text { Périmètre } \\
\text { thoracique }\end{array}$ & $\begin{array}{c}\text { Poids moyen } \\
\text { mâles }\end{array}$ & $\begin{array}{c} \pm \text { kg* } \\
(95 \%)\end{array}$ & $\begin{array}{l}\text { Poids moyen } \\
\text { femelles }\end{array}$ & $\begin{array}{c} \pm \mathbf{k g}^{*} \\
(95 \%)\end{array}$ \\
\hline 0,7 & 30,56 & 3,71 & 32,37 & 3,67 \\
\hline 0,75 & 35,34 & 3,08 & 36,71 & 3,04 \\
\hline 0,80 & 40,68 & 2,56 & 41,62 & 2,51 \\
\hline 0,85 & 46,58 & 2,13 & 47,08 & 2,07 \\
\hline 0,90 & 53,04 & 1,80 & 53,09 & 1,74 \\
\hline 0,95 & 60,05 & 1,56 & 59,67 & 1,50 \\
\hline 1,00 & 67,63 & 1,38 & 66,80 & 1,33 \\
\hline 1,05 & 75,76 & 1,27 & 74,49 & 1,23 \\
\hline 1,10 & 84,44 & 1,19 & 82,74 & 1,16 \\
\hline 1,15 & 93,69 & 1,14 & 91,54 & 1,12 \\
\hline 1,20 & 103,49 & 1,13 & 100,91 & 1,11 \\
\hline 1,25 & 113,85 & 1,18 & 110,83 & 1,16 \\
\hline 1,30 & 124,77 & 1,32 & 121,31 & 1,29 \\
\hline 1,35 & 136,25 & 1,58 & 132,34 & 1,52 \\
\hline 1,40 & 148,28 & 1,95 & 143,94 & 1,87 \\
\hline 1,45 & 160,87 & 2,43 & 156,09 & 2,33 \\
\hline 1,50 & 174,02 & 3,01 & 168,80 & 2,90 \\
\hline
\end{tabular}

* Fluctuation calculée pour chaque prédiction. Le calcul de l'intervalle de confiance n'est valable que pour les valeurs de périmètres thoraciques pris indépendamment les uns des autres 


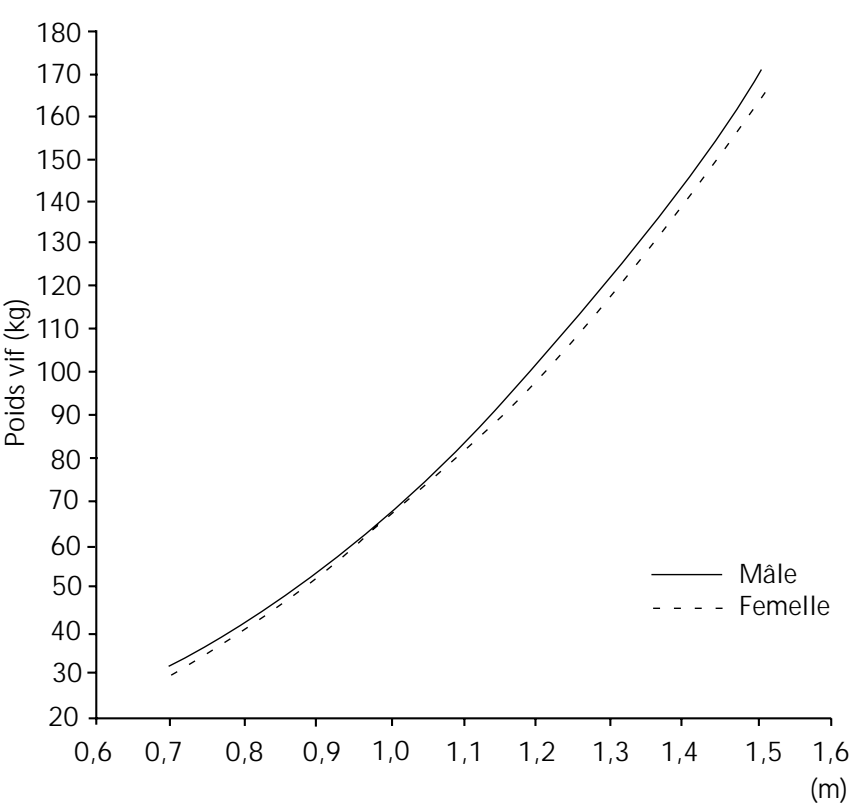

Figure 3 : courbes moyennes du poids des dromadaires mâles et femelles en fonction du périmètre thoracique.

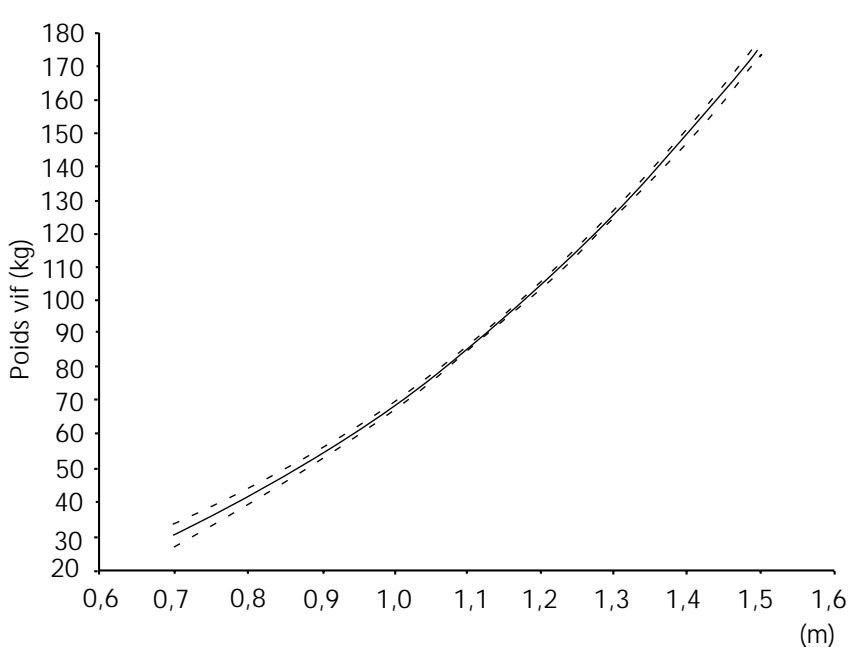

Figure 4 : courbe moyenne du poids et intervalle de confiance des dromadaires mâles en fonction du périmètre thoracique.

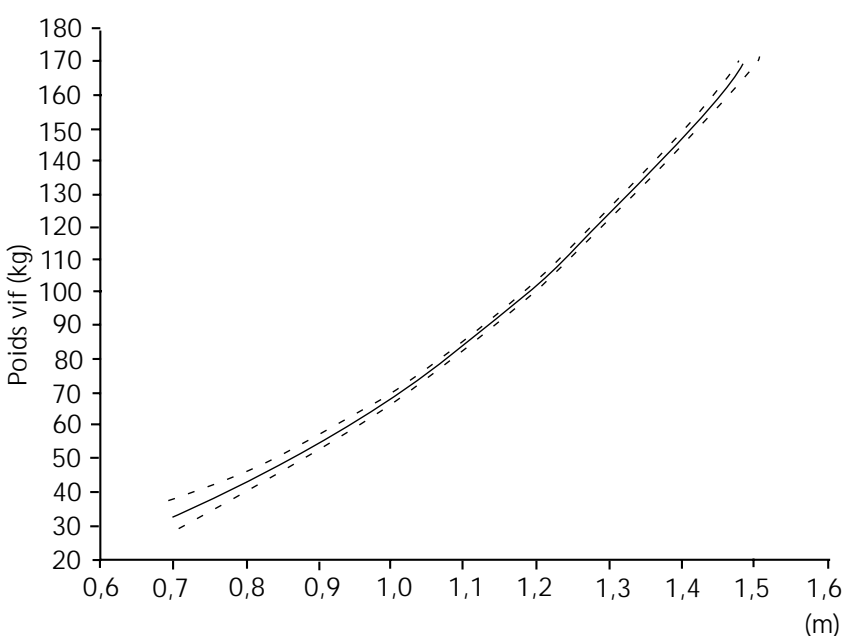

Figure 5 : courbe moyenne du poids et intervalle de confiance des dromadaires femelles en fonction du périmètre thoracique.
Le modèle sous-estime légèrement les poids des dromadaires à partir de $150 \mathrm{~kg}$ (non montré). Cela peut être causé par la faiblesse du nombre de pesées de plus de $150 \mathrm{~kg}$. Ce poids correspondait à une limite expérimentale liée à l'appareil de mesure et surtout aux difficultés de contention des chamelons âgés de plus de six mois.

\section{Croissance pondérale}

Le rythme au minimum mensuel du recueil des données ayant introduit une erreur expérimentale sur la précision des dates de naissance des chamelons (mémoire imprécise des éleveurs), il n'a pas été possible de tenir compte des mesures prises sur les animaux de moins de 15 jours, période pendant laquelle la croissance est très rapide.

Le manque de pesées de plus de $150 \mathrm{~kg}$ a contraint à limiter la modélisation de la croissance pondérale aux six premiers mois d'âge. Cette contrainte ne s'était pas imposée dans la modélisation de la barymétrie car, pour des raisons physiologiques, la variabilité du poids selon les mensurations HG ou PT est beaucoup moins importante que celle de la croissance selon l'âge qui subit des effets de facteurs environnementaux et humains. D'autre part, pour pallier aux difficultés d'estimation des paramètres de ce modèle, seuls les chamelons qui fournissaient au moins trois observations de poids ont été inclus.

La description du plan d'observation de la modélisation de la croissance des chamelons de 15 à 180 jours est présentée dans le tableau IV. La variable poids a été transformée en log pour diminuer l'hétérogénéité de la variance. Les paramètres du modèle estimés avec la méthode du maximum de vraisemblance restreinte sont présentés dans le tableau $\mathrm{V}$.

Ce modèle a permis le calcul des poids à âges types (tableau VI, figures 6 à 8) et des gains moyens quotidiens (tableau VII) utiles pour comparer les vitesses de croissance des chamelons.

Le modèle de croissance pondérale n'a pas révélé d'effet de la vermifugation de la mère dans les 500 jours précédant la mise bas.

\section{DISCUSSION}

\section{Formules barymétriques}

La hauteur au garrot étant fortement corrélée au périmètre thoracique $(\mathrm{r}=0,9 ; \mathrm{p}<0,05)$ la formule barymétrique validée n'a tenu compte que d'une mensuration, le périmètre thoracique. Elle ramène le travail de prédiction du poids à la prise d'une seule mesure. Ce travail de simplification qui constitue l'objectif de la barymétrie permettra à l'avenir d'estimer facilement le poids des chamelons de moins de $175 \mathrm{~kg}$ (moins de 1,5 an environ) en évitant les problèmes de contention ou de limite d'appareillage de mesure comme ce fut le cas dans cette étude.

Le sexe est apparu comme un facteur de variation significatif $(\mathrm{p}<0,01)$ du modèle retenu. L'observation des courbes de prédiction du poids des mâles et des femelles a montré un croisement pour $0,90 \mathrm{~m}$ de périmètre thoracique (tableau III) correspondant à environ 15 jours d'âge (tableau VI). Cela démontre une croissance allométrique liée au sexe du chamelon : à périmètre thoracique égal, les femelles ont été légèrement plus lourdes que les mâles avant l'âge de 15 jours (jusqu'à 6 p. 100 pour $\mathrm{PT}=0,7 \mathrm{~m}$ ), puis le phénomène s'est inversé (jusqu'à 3 p. 100 pour $\mathrm{PT}=1,5$ ). La constatation, à cage thoracique de même format, de femelles plus lourdes que les mâles avant l'âge de 15 jours est à rapprocher des études citées par Wilson (15) rapportant un poids plus élevé des femelles à la naissance. 
Growth performances of camel calves in Niger

\section{Tableau IV}

Plan d'observation de la modélisation de la croissance des dromadaires de 15 à 180 jours

$\mathrm{Nb}$. de mesures

Saison favorable

Saison défavorable

Saison favorable

Saison défavorable

Vermifugation

Mâle
Femelle

Femelle
Saison favorable Saison défavorable Saison favorable Saison défavorable
117

50

101

78

74

12

76

24

532
Nb. d'animaux

\section{9}

14

24

21

18

3

18

6

133

Tableau V

Estimation des paramètres du modèle mixte log(Poids vif)-Age par la méthode Reml

\begin{tabular}{|c|c|c|c|c|c|c|}
\hline Paramètres & Valeur & Ecart-type & ddl1 & ddI2 & Test F & $\mathbf{p}$ \\
\hline \multicolumn{7}{|l|}{ Effets fixes } \\
\hline Constante & 4,383 & 0,024 & 1 & 396 & 36373,95 & $<0,0001$ \\
\hline Age & 0,130 & 0,003 & 1 & 396 & 1121,36 & $<0,0001$ \\
\hline $\mathrm{Age}^{2}$ & $-0,016$ & 0,001 & 1 & 396 & 133,04 & $<0,0001$ \\
\hline Sexe & $-0,013$ & 0,012 & 1 & 110 & 2,61 & 0,1091 \\
\hline Saison & $-0,044$ & 0,013 & 1 & 110 & 11,24 & 0,0011 \\
\hline Vermifugation & 0,006 & 0,013 & 1 & 110 & 0,36 & 0,5517 \\
\hline Age $x$ Vermifugation & 0,004 & 0,002 & 1 & 396 & 2,79 & 0,0958 \\
\hline \multicolumn{7}{|l|}{ Effets aléatoires corrélés } \\
\hline \multicolumn{7}{|l|}{$\mathrm{N}$ iveau animal } \\
\hline$\sigma$ (constante) & 0,129 & & & & & \\
\hline$\sigma$ (âge) & 0,019 & & & & & \\
\hline$\sigma$ (constante, âge) & $-0,106$ & & & & & \\
\hline \multicolumn{7}{|l|}{$\mathrm{N}$ iveau éleveur } \\
\hline$\sigma$ (constante) & 0,081 & & & & & \\
\hline$\sigma$ (âge) & 0,011 & & & & & \\
\hline$\sigma$ (constante, âge) & $-0,613$ & & & & & \\
\hline$\sigma$ (erreur résiduelle) & 0,063 & & & & & \\
\hline
\end{tabular}

$\sigma(\mathrm{a})$ : écart-type du paramètre aléatoire a

$\sigma(a, b)$ : covariance paramètres aléatoires a et b

dd11 : nombre de degrés de liberté du numérateur

dd12 : nombre de degrés de liberté du dénominateur 


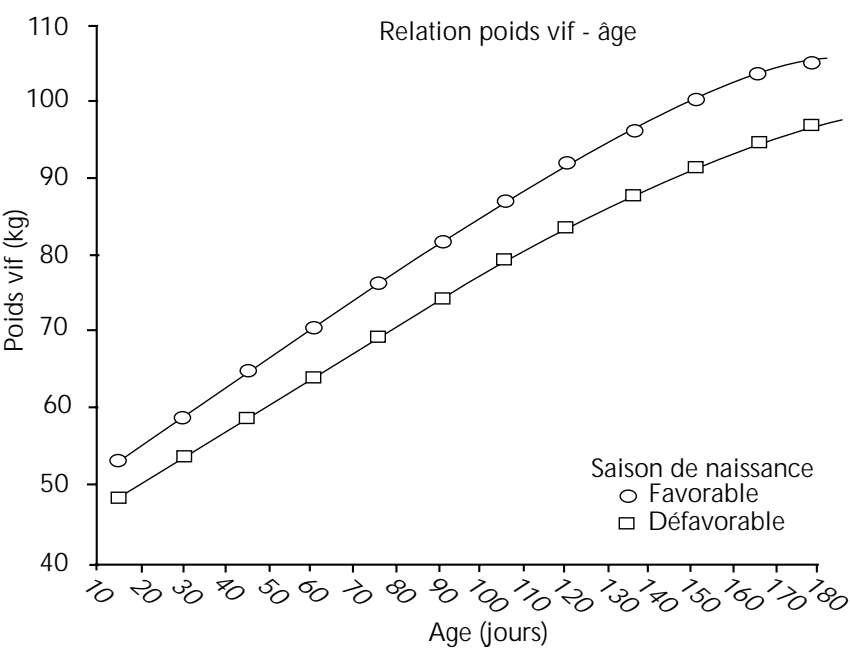

Figure 6 : courbe moyenne de croissance des dromadaires de 15 à 180 jours selon la saison de naissance.
Performances de croissance du chamelon au Niger

Tableau VI

Poids moyens des dromadaires de 15 à 180 jours selon l'âge et la saison de naissance

\begin{tabular}{ccccc} 
& \multicolumn{2}{c}{ Saison favorable } & \multicolumn{2}{c}{ Saison défavorable } \\
$\begin{array}{c}\text { Age } \\
\text { (jours) }\end{array}$ & $\begin{array}{c}\text { Poids moyen } \\
\mathbf{( k g )}\end{array}$ & $\begin{array}{c}\mathbf{\mathbf { k g }} \\
\mathbf{( 9 5 \% )}\end{array}$ & $\begin{array}{c}\text { Poids moyen } \\
\mathbf{( k g )}\end{array}$ & $\begin{array}{c}\mathbf{\mathbf { k g }} \\
\mathbf{( 9 5 \% )}\end{array}$ \\
\hline 15 & 53,36 & 1,730 & 48,52 & 1,845 \\
30 & 59,09 & 1,713 & 53,73 & 1,833 \\
45 & 64,91 & 1,709 & 59,03 & 1,832 \\
60 & 70,74 & 1,715 & 64,33 & 1,837 \\
75 & 76,55 & 1,725 & 69,62 & 1,846 \\
90 & 82,11 & 1,738 & 74,66 & 1,856 \\
105 & 87,36 & 1,752 & 79,44 & 1,868 \\
120 & 92,20 & 1,766 & 83,85 & 1,881 \\
135 & 96,64 & 1,783 & 87,88 & 1,895 \\
150 & 100,38 & 1,804 & 91,29 & 1,913 \\
165 & 103,44 & 1,831 & 94,07 & 1,936 \\
180 & 105,85 & 1,867 & 96,25 & 1,967 \\
& & & & \\
\end{tabular}

Tableau VII

Gain moyen quotidien ( $\mathrm{Gmq}$ ) des dromadaires selon la saison de naissance

\begin{tabular}{lccc}
$\begin{array}{l}\text { Périodes } \\
\text { (jours) }\end{array}$ & \multicolumn{2}{c}{ G mq (g/jour) } & $\begin{array}{c}\text { Différence } \\
\text { (g/jour) }\end{array}$ \\
\cline { 2 - 3 } & Saison favorable & Saison défavorable & \\
\hline $15-30$ & 382 & 347 & 35 \\
$30-60$ & 388 & 353 & 35 \\
$60-90$ & 379 & 344 & 35 \\
$90-120$ & 337 & 306 & 31 \\
$120-180$ & 227 & 207 & 20 \\
$15-180$ & 318 & 289 & 29 \\
\hline
\end{tabular}

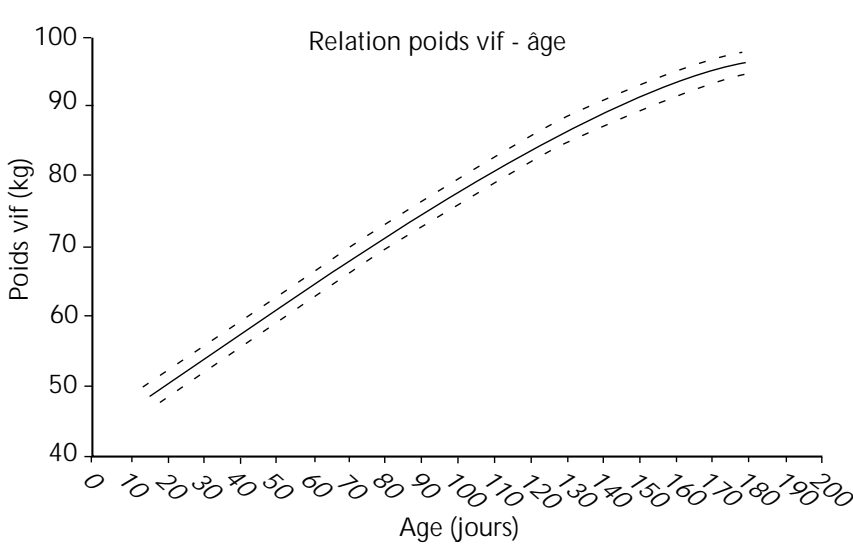

Figure 8 : courbe moyenne de croissance des dromadaires de 15 à 180 jours en saison de naissance défavorable et intervalle de fluctuation à 95 p. 100.

\section{Performances de croissance des jeunes}

Selon les auteurs, le sexe des chamelons peut influencer (2) ou ne pas influencer (5) la croissance des chamelons. L'étude menée n'a pas révélé d'effet du sexe sur la croissance des chamelons de 15 à 180 jours.

En revanche, la croissance des chamelons âgés de 15 à 180 jours a été influencée par la saison de naissance $(p<0,01)$ : ceux des chamelons qui sont nés en saison des pluies et en saison sèche froide ont été, au même âge, 10 p. 100 plus lourds que ceux qui sont nés pendant la saison sèche chaude (ces derniers ont représenté respectivement 20 et 5 p. 100 du total annuel de naissances des troupeaux Touareg et Toubou ; figure 9). Des études se sont déjà intéressées à l'effet de la saison sèche sur la vitesse de croissance et, selon le cas, cet effet a été mis (4) ou non (5) en évidence. Il est intéressant de noter que, dans cette étude, la saison sèche a été partagée en deux, la saison sèche froide s'étant révélée favorable à 


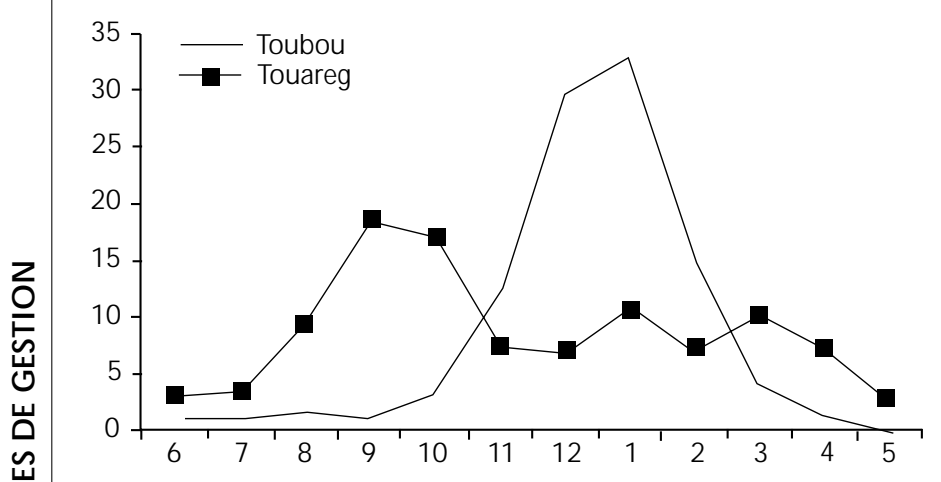

Figure 9 : distribution mensuelle des naissances en fonction de l'ethnie de l'éleveur.

la croissance des jeunes au même titre que la saison des pluies. Il est probable que pendant cette période les conditions alimentaires ont été satisfaisantes pour la production laitière de la chamelle ; ce n'est qu'à partir de mars que la diminution des quantités de fourrages disponibles, alliée à leur dessèchement rapide induit par la hausse des températures, a privé les chamelles de nutriments favorables à une bonne production laitière.

Le poids des chamelons a doublé entre les âges de 15 et 180 jours passant respectivement en saisons favorable et défavorable de $53,36 \pm 1,73 \mathrm{~kg}$ à $105,8 \pm 1,87 \mathrm{~kg}$ et de $48,52 \pm 1,84 \mathrm{~kg}$ à 96,25 $\pm 1,97 \mathrm{~kg}$ (tableau VI). Le gain moyen quotidien durant cette période a varié de $318 \mathrm{~g} / \mathrm{j}$ en saison favorable à $289 \mathrm{~g} / \mathrm{j}$ en saison défavorable (tableau VII). Ces vitesses de croissance relativement faibles s'accordent avec celles qui sont rencontrées dans d'autres systèmes d'élevage traditionnels africains $(4,11)$. Elles s'expliquent principalement par la compétition existant entre l'homme et le chamelon pour la consommation du lait de la chamelle (4). Dans la zone d'étude, la quantité moyenne traite a été estimée sur 12 chamelles à 2,3 1 de lait par jour au cours des premiers mois de la naissance, ce qui correspond à environ 50 p. 100 de la production totale admise pour la chamelle au Niger (14). L'importance de cette concurrence ne laisse pas la possibilité aux chamelons d'exprimer tout leur potentiel génétique comme cela peut être le cas en élevage intensif (3).
Le gain moyen quotidien (gmq) a été stable entre 15 et 90 jours ( $383 \mathrm{~g} / \mathrm{j}$ pour les chamelons nés en saison favorable et $348 \mathrm{~g} / \mathrm{j}$ pour ceux nés en saison sèche chaude), puis a diminué progressivement pour atteindre respectivement dans les deux groupes 227 et $207 \mathrm{~g} / \mathrm{j}$ entre 120 et 180 jours (tableau VII). Le bénéfice de l'effet de la saison sur les gmq (+ 10 p. 100) a été constant chez les chamelons âgés de 15 et 180 jours.

Le statut nutritionnel et sanitaire de la mère peut influencer le poids et la santé du chamelon (12). La vermifugation de la mère dans les 500 jours précédant la mise bas était utilisée dans cette étude comme un indicateur d'un bon encadrement sanitaire et nutritionnel de la mère pendant la préparation à la saillie et pendant la gestation. Ce facteur était d'un intérêt majeur, la saison et le sexe étant des cofacteurs de variation dont il fallait tenir compte pour évaluer l'impact de la vermifugation de la mère sur la croissance du chamelon. Les tests effectués n'ont pas mis en évidence d'effet de cette vermifugation des mères au cours des 500 jours avant la mise bas. L'étude de ce facteur de vermifugation mérite d'être approfondie, en réduisant sans doute la période de 500 jours d'influence de la vermifugation.

\section{CONCLU SION}

Le poids des chamelons a été estimé à l'aide d'une formule barymétrique basée sur des mesures de périmètre thoracique comprises entre 0,7 et 1,5 m. Cette étude a mis en évidence une croissance allométrique liée au sexe du chamelon.

Des difficultés expérimentales liées aux instruments de mesure et à la contention des animaux n'ont permis de modéliser la croissance des chamelons qu'entre les âges de 15 et 180 jours. Les formules barymétriques permettront de lever ces contraintes pour des chamelons de moins de $175 \mathrm{~kg}$ (âgés de moins de 18 mois).

La saison de naissance a influencé significativement la croissance entre 15 et 180 jours : les chamelons nés en saison des pluies et en saison sèche froide ont été en moyenne 10 p. 100 plus lourds et ont grandi 10 p. 100 plus vite que ceux nés en saison sèche chaude. Les vitesses de croissance faibles (respectivement 318 et $289 \mathrm{~g} / \mathrm{j}$ en saisons favorable et défavorable) étaient caractéristiques des systèmes d'élevage extensifs où l'homme et le chamelon se partagent la production laitière de la mère.

\section{BIBLIO GRAPHIE}

1. ABBAS B., LE HORGNE J.M., SAINT-MARTIN G., 1992. Association of a short term inquiry and a continuous survey in order to study the camel farming systems in Eastern Sudan. In: 2nd International Symposium on the Study of Livestock Farming Systems in Research and Development Framework, 11-12 September 1992, Saragossa, Spain, 5 p.

2. BURGEM EISTER R., 1975. Elevage de chameaux en Afrique du Nord Eschborn, Germany, Gtz, $86 \mathrm{p}$.

3. DEGEN A.A., ELIAS E., KAM M., 1987. A preliminary report on the energy intake and growth rate of early weaned camel (Camelus dromedarius) calves. Anim. Prod., 45: 301-306.

4. FIELD C.R., 1979. Camel growth and milk production in Marsabit district, Northern Kenya, provisionnal report no. 6. In: IFS Camels, IFS, Stockolm, Sweden, p. 215-240.
5. HULSEBU SCH C., 1999. Immunoglobulin $G$ status of camels during 6 months post natum. Stuttgart, Germany, Magraf Verlag/University of Hohenheim, 148 p. (Hohenheim Tropical Agricultural Series)

6. LAIRD N.M., WARE J.H., 1982. Random-effects models for longitudinal data. Biometrics, 38: 963-974.

7. MINISTERE DE L'AGRICULTURE ET DE L'ELEVAGE, 1997. Filières agropastorales, filières d'élevage. In : Secteur rural du Niger, PASA, Niamey, Niger, p. 73-96.

8. PINHEIRO J.C., BATES D.M., 2000. Mixed effects models in $\mathrm{S}$ and Splus. Berlin, Germany, Springer, $552 \mathrm{p}$.

9. PLANCHENAULT D., 1984. Production cameline - Résultats zootechniques. Projet de développement de l'élevage dans le Niger Centre-Est. Maisons-Alfort, France, Gerdat-lemvt, 213 p. 
10. PLAN CHENAULT D., SAHUT C., 1989. Pikbeu : manuel d'utilisation. Maisons-Alfort, France, lemvt, $85 \mathrm{p}$.

11. RICHARD D., HOSTE C., PEYRE DE FABREgUES B., 1984. Les paramètres de production. In : Le dromadaire et son élevage. MaisonsAlfort, France, Cirad-lemvt, p. 77-104. (Etudes et synthèses)

12. SIM PKIN S.P., 1985. The effects of disease as constraints to camel production in Northern Kenya. In: Camel diseases and productivity in the arid lands of Northern Kenya. Nairobi, Kenya, U nited Nations Educational, Social and Cultural Organization, p. 77-160. (IPAL Technical Report No. E-7)
13. S-PLUS, 1998. Data analysis products division. Seatle, WA, USA, Mathsoft

14. STEIN M ETZ P., 1995. Le lait de chamelle enfin apprivoisé. In : Rapport d'activités, projet camelin, phase 1 , annexe $n^{\circ} 5$. Montpellier, France, Cirad-emvt/M inistère de l'Agriculture et de l'Elevage, Niger, $150 \mathrm{p}$.

15. WILSON R.T., 1992. Factors affecting weight and growth in onehumped camels. Proc. 1st. International Camel Conference, Dubai, United Arab Emirates, 2-6 February 1992. Newmarket, Suffolk, UK, R\&W Publishers, p. 309-312.

\section{Summary}

Pacholek X., Lancelot R., Lesnoff M., Messad S. Growth performance of camel calves raised in the pastoral zone of $\mathrm{N}$ iger

From 1992 to 1996, camel calves under one year of age from 20 herds and traditionally raised in the pastoral zone of Niger were individually monitored for zootechnical data. The study aimed at establishing a simple barymetric formula to estimate the weight of camel calves and modelizing weight gain taking into account some variation factors. Allometric data (live weight, height at withers, chest girth) were analyzed by the linear mixed model method. A barymetric formula was established from the measure of chest girth (CG) comprised between 0.7 and $1.5 \mathrm{~m}$. Sex appeared as a significant factor of variation $(P<0.01)$ : with the same $C G$, females were heavier than males at birth, then from $C G=0.9 \mathrm{~m}$ it was reversed. Predicted live weights varied between $30.56 \pm 3.71$ and $174.02 \pm 3.01 \mathrm{~kg}$ in males and $32.37 \pm 3.67$ to 168.80 $\pm 2.90 \mathrm{~kg}$ in females. A growth model was calculated for camel calves aged 5 to 180 days. No sex effect or vermifugation effect of the mother in the 500 days before parturition (sign of nutrition and health states) were found. O n the other hand, camel calves born during the rainy season or at the beginning of the dry season were $10 \%$ heavier and grew $10 \%$ faster than those born during the hot dry season $(P<0.01)$. Average daily gains were low with 318 and $289 \mathrm{~g} / \mathrm{d}$ in the favorable and unfavorable seasons, respectively, typical of extensive farming systems.

Key words: Dromedary - Young animal - Growth - Body measurement - W eight - Productivity - Niger.

\section{Resumen}

Pacholek X., Lancelot R., Lesnoff M., Messad S. Rendimientos de crecimiento de los camellos jóvenes criados en la zona pastoril nigeriana

De 1992 a 1996 se llevó a cabo un seguimiento zootécnico individual sobre los camellos jóvenes, de menos de un año, criados tradicionalmente en 20 hatos en la zona pastoril nigeriana. El estudio pretendía establecer una fórmula barimétrica simple para la estimación del peso de los camellos, así como realizar modelos del crecimiento ponderal, teniendo en cuenta eventuales factores de variación. Los datos alometricos (peso vivo, altura a la cruz, perímetro torácico), fueron analizados según la metodología de los modelos lineares mixtos. Una formula barimétrica se estableció a partir de la medida de los perímetros torácicos (PT), comprendidos entre 0,7 y $1,5 \mathrm{~m}$. El sexo se presentó como un factor de variación significativo ( $p<0,01)$ : a perímetro torácico igual, las hembras nacen más pesadas, este fenómeno se invierte luego, a partir de $\mathrm{PT}=0,9 \mathrm{~m}$. Los pesos vivos predichos variaron entre $30,56 \pm 3,71$ y $174,02 \pm 3,01 \mathrm{~kg}$ para los machos y 32,37 $\pm 3,67$ y $168,80 \pm 2,90 \mathrm{~kg}$ para las hembras. Se calculó un modelo de crecimiento para los camellos con edades entre 15 y 180 días. No se demostró ningún efecto del sexo o de la desparasitación de la madre en los 500 días precedentes al parto (indicador del estado sanitario y nutricional). Sin embargo, los camellos nacidos durante la estación de lluvias y al inicio de la estación seca fueron $10 \%$ más pesados y crecieron $10 \%$ más rápido que los camellos nacidos durante la estación cálida $(p<0,01)$. Las ganancias de peso cotidianas fueron bajas: 318 y 289 g/día respectivamente durante las estaciones favorables y desfavorables, características de los sistemas de crianza intensivos.

Palabras clave: Dromedario - Animal joven - Crecimiento Medición del cuerpo - Peso - Productividad - N iger. 